\title{
No association of the SNP rs1048261 within 3'UTR of HSPB7 with cardiovascular morbidity in patients with psoriasis; a possible effect on miRNA-mediated translational regulation
}

\author{
Pavel Hruska1*, Filip Zlamal ${ }^{1,3}$, Vladimir Vasku² and Julie Bienertova-Vasku ${ }^{1,3}$ \\ ${ }^{1}$ Department of Pathological Physiology, Faculty of Medicine, Masaryk University, Kamenice 5, Building A18, 62500 Brno, Czech Republic \\ ${ }^{2}$ First Department of Dermatology, St. Anne’s University Hospital Brno, Pekařská 53, 65691 Brno, Czech Republic \\ ${ }^{3}$ Research Centre for Toxic Compounds in the Environment, Kamenice 5, Building A29, 62500 Brno, Czech Republic
}

\begin{abstract}
Background: Previous studies have shown an increased risk of cardiovascular diseases in patients with psoriasis. It has also been known that abnormal levels of microRNA (miRNA) contribute to the pathogenesis of psoriasis and its comorbidities. Moreover, single nucleotide polymorphisms (SNPs) within miRNA genes or 3'UTR of the miRNA target genes can alter gene expression and thus contribute to the pathogenesis of the disease.

Objectives: Assess SNP rs1048261 within the 3'UTR of HSPB7 in a large cohort ( $\mathrm{n}=558)$ of psoriatic patients of Central European Caucasian origin and evaluate its possible role in the increased risk of cardiovascular disease (CVD) in psoriasis patients.

Methods: This study included 558 patients diagnosed with psoriasis; 30\% of the patients had a personal history of CVD. In silico analysis was used to determine a candidate SNP which is associated with a potential impairment of miRNA-mediated translational regulation. The chosen SNP rs1048261 was genotyped using PCR-RFLP and all statistical analyses were performed using statistical software R.

Results: Possible interactions of the genotype and personal history of CVD were tested, but no significant association was found. Subsequently, the clinical subtypes of psoriasis (plaque psoriasis, pustular psoriasis and guttate psoriasis) as well as age at psoriasis onset were analyzed to associate the genotype with CVD, but neither association was found to be statistically significant. Finally, the logistic regression model was created to test any possible associations with the personal history of CVD, but all results were inconclusive.

Conclusions: Although our study did not provide any significant association of the SNP rs1048261 with cardiovascular morbidity, we suggest investigating this polymorphism in a cohort of patients with properly diagnosed cardiovascular diseases independently of psoriasis. Also, the validation of miRNA-mRNA interaction and SNP rs1048261 involvement needs to be studied.
\end{abstract}

\section{Introduction}

Psoriasis is a chronic inflammatory skin disease with an estimated prevalence of approximately $3 \%$ in the Caucasian population [1]. The disease is characterized by well-defined red scaly plaques which appear on the skin as a result of abnormal proliferation and differentiation of keratinocytes and inflammation [2]. Apart from the skin manifestation, psoriasis is a complex disease and is therefore associated with a variety of comorbidities, e.g. psoriatic arthritis, diabetes mellitus, obesity, hypertension [3] and - most importantly - with an increased risk of cardiovascular disease [4-8].

A common feature in inflammatory pathways, including inflammation in psoriasis, is the generation of oxidative stress and the production of reactive oxygen species (ROS) which may cause oxidative damage to lipids and proteins in cells [9]. However, cells are protected by evolutionary conserved defense and repair mechanisms to neutralize these damages. Any imbalance in these mechanisms may therefore increase the amount of ROS or unrepaired damage in cells and thus lead to a pathological state of a disease. Such a defect may be considered as a risk factor of CVD in psoriasis [10-11].
One of the cellular defense responses to oxidative damage is an induced expression of heat shock proteins (Hsp) which are able to repair denatured proteins and promote their degradation [12]. In this study we focused on a cardiac specific member of the small Hsp family, HSPB7. HSPB7, also known as cvHsp [13], is characterized by its relatively smaller molecular weight $18,6 \mathrm{kDa}$ and a highly conserved $a$-crystallin domain, which is important for its chaperon-like activity $[14,15]$. This abundant protein is selectively expressed in myocardial muscle cells, but it was also found to be expressed at lower levels in skeletal muscle and adipose tissue [13]. Its molecular architecture displays properties of the small Hsp family and seems to be importantly implicated in cardiac stress response [16]. Although its biological

Correspondence to: Pavel Hruska, Department of Pathological Physiology, Faculty of Medicine, Masaryk University, Kamenice 5, Building A18, 62500 Brno, Czech Republic, Tel: +420 736118 600; E-mail: 394282@mail.muni.cz

Key words: HSPB7, SNP, rs1048261, miRNA, microRNA, psoriasis, cardiovascular disease, comorbidity

Received: March 05, 2016; Accepted: April 08, 2016; Published: April 13, 2016 
Hruska P (2016) No association of the SNP rs1048261 within 3’UTR of HSPB7 with cardiovascular morbidity in patients with psoriasis; a possible effect on miRNA-mediated translational regulation

function is not yet clear, HSPB7 seems to have a protective function, preventing the misfolding or refolding of denatured cytoskeletal proteins under acute stress during myocardial infarction. However, high levels of HSPB7 are associated with an increased risk of acute coronary syndrome [17].

MicroRNAs (miRNAs) are small $\sim 21 \mathrm{nt}$ long molecules of non-coding RNA that can regulate gene expression at the posttranscriptional level by imperfect base-pairing to the 3'UTR of the target mRNA [18]. For the target recognition and regulation, the sequence of a miRNA defined as the first 2-8nt counting from the beginning of the 5' end toward the 3' end seems to be important [19]. Recent research has indicated the involvement of MiRNAs in the pathology of various diseases including psoriasis [20,21] and cardiovascular diseases [22,23]. However, not only changes in miRNA expression are associated with disease pathologies. Recent studies have shown that SNPs within the seed region or target sequence in the 3'UTR of the target gene can create, disturb or alter miRNA-target interactions which may lead to a disruption of its biological function, thus affecting the development and phenotype of many diseases [24,25].

In this study we aimed to identify whether the SNP rs1048261 within the 3'UTR of HSPB7, which may disrupt the mechanism of miRNAs' regulation of HSPB7 expression, is associated with the increased cardiovascular morbidity in patients with psoriasis.

\section{Materials and methods}

\section{Subjects}

The cohort of psoriasis patients ( $n=558$; sex ratio $M / F=244 / 314$; mean age $49.7 \pm 16.4$; mean age at psoriasis onset $26.9 \pm 15.9$ ) was collected at the First Department of Dermatology, St. Anne's University Hospital, Brno, Czech Republic. All participating patients were of Caucasian origin. Individuals were clinically diagnosed with psoriasis and a careful description of family and personal history of psoriasis and cardiovascular diseases was documented. All patients were classified into three clinical subtypes (plaque psoriasis $n=428$; pustular psoriasis $\mathrm{n}=22$; guttate psoriasis $\mathrm{n}=106$ ) according to phenotype based on criteria defined by Griffiths et al. [26]. 30\% of all patients suffered from CVD.

The study was approved by the Ethics Committee of Medical Experiments on Human Subjects of Masaryk University, Brno, Czech Republic and was performed in accordance with the ethical standards of the Helsinki Declaration guidelines. All participants provided written informed consent which was subsequently archived.

\section{Bioinformatics}

Databases MirSNP [27] and miRNASNP2 [24] were searched for possible miRNA-related SNPs within HSPB7 (last accessed March 16, 2016).

The query results were then filtered to meet the following conditions:

- $\quad$ minor allele frequency (MAF) higher than 5\%

- $\quad$ average expression of associated miRNA of at least 10 reads per million (RPM)

- $\quad$ SNP in the genome-wide association study (GWAS) linkage disequilibrium (LD) region

Only rs1048261 SNP met the listed conditions and was then analyzed in a large cohort of Central European patients with psoriasis as a possible risk factor of CVD. Information about rs1048261 SNP and associated miRNAs is listed in Figure 1.

\section{Genotyping}

Analyzed genomic DNA was extracted from peripheral blood

\begin{tabular}{|c|c|c|c|}
\hline SNP & \multirow{2}{*}{$\begin{array}{l}\text { Gene } \\
\text { HSPB7 }\end{array}$} & Location & \multirow{2}{*}{$\begin{array}{l}\text { MAF (EUR) } \\
A=0.32 / 1,006 \text { (1000GENOMES) }\end{array}$} \\
\hline \begin{tabular}{l|l} 
rs1048261 & HS \\
\end{tabular} & & Chr1:16340951 & \\
\hline miRNA & $\begin{array}{l}\text { miRNA } \\
\text { exp. }^{\star}\end{array}$ & Effect & miRNA/target duplex with SNP \\
\hline hsa-miR-15a-5p & 168.55 & $\begin{array}{l}\text { Loss of binding } \\
\text { site }\end{array}$ & 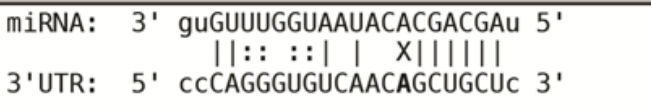 \\
\hline hsa-miR-15b-5p & 473.87 & $\begin{array}{l}\text { Loss of binding } \\
\text { site }\end{array}$ & 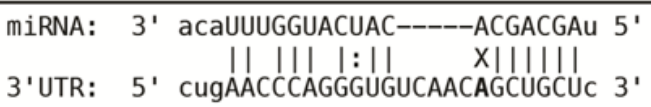 \\
\hline hsa-miR-16-5p & 668.25 & $\begin{array}{l}\text { Loss of binding } \\
\text { site }\end{array}$ & 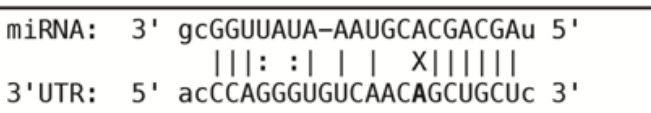 \\
\hline hsa-miR-195-5p & 37.74 & $\begin{array}{l}\text { Loss of binding } \\
\text { site }\end{array}$ & 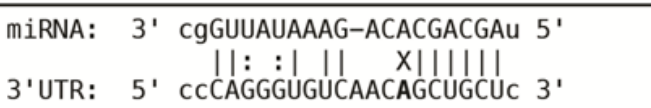 \\
\hline hsa-miR-424-5p & 158.86 & $\begin{array}{l}\text { Loss of binding } \\
\text { site }\end{array}$ & 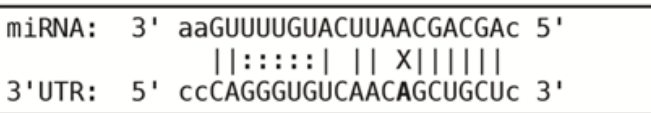 \\
\hline hsa-miR-497-5p & 22.89 & $\begin{array}{l}\text { Loss of binding } \\
\text { site }\end{array}$ & 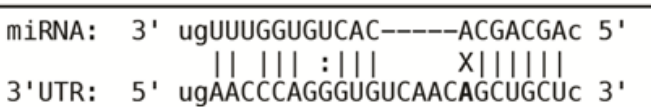 \\
\hline hsa-miR-1267 & 0.01 & $\begin{array}{l}\text { Gain of binding } \\
\text { site }\end{array}$ & 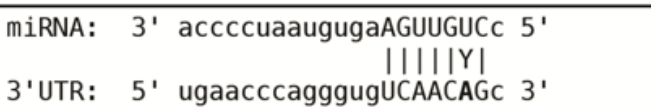 \\
\hline
\end{tabular}


Hruska P (2016) No association of the SNP rs1048261 within 3’UTR of HSPB7 with cardiovascular morbidity in patients with psoriasis; a possible effect on miRNA-mediated translational regulation

using a standard technique with Proteinase K. The rs1048261 in 3'UTR of HSPB7 was detected by using polymerase chain reaction and restriction fragment length polymorphism (PCR-RFLP) analysis. The amplified fragment of $219 \mathrm{bp}$ was generated by using the following primers: forward 5'-GTGCTGTGCTTTGTCACTGA-3' and reverse 5'-CCAGGGTTAAGGCTGATGGA-3'. PCR was performed using $0.1 \mu \mathrm{g}$ of genomic DNA as a template under the following conditions: denaturation process for $10 \mathrm{~min}$ at $95^{\circ} \mathrm{C} ; 32$ cycles of denaturation for $30 \mathrm{~s}$ at $95^{\circ} \mathrm{C}$, annealing for $20 \mathrm{~s}$ at $60^{\circ} \mathrm{C}$ and extension for $30 \mathrm{~s}$ at $72^{\circ} \mathrm{C}$; and final extension step for $5 \mathrm{~min}$ at $72^{\circ} \mathrm{C}$, followed by a cooling process to $15^{\circ} \mathrm{C}$. The PCR product $(10 \mu \mathrm{l})$ was then digested for RFLP analysis using 3 units of the restriction enzyme PvuII with Buffer G. The digestion was performed for 12 hours at $37^{\circ} \mathrm{C}$ and the products were then separated on $4 \%$ agarose gel stained with ethidium bromide. Fragment length was 140-79 bp in the case of the A allele, and 50-90-79 $\mathrm{bp}$ in the case of the $\mathrm{T}$ allele (alleles are reported in forward orientation to the genome).

\section{Statistics}

Statistical software $\mathrm{R}$ (version 3.2.1) was used to perform all statistical analyses. Continuous variables were expressed as mean \pm $\mathrm{SD}$ and categorical variables as percentages. The asymptotic Pearson's $\chi^{2}$ and Fisher's exact test was used to test the Hardy-Weinberg equilibrium. The relationship between categorical variables was tested by Pearson's $\chi^{2}$ test of independence. A non-parametric Kruskal-Wallis test was used for intergroup comparisons while the Wilcoxon rank sum test with Benjamini and Hochberg p-value adjustments was used for pairwise comparisons where appropriate. A logistic regression model was created with personal CVD history as a dependent variable and HSPB7 genotypes, sex, age at psoriasis onset and clinical subtypes of psoriasis as independent variables. This model was then reduced by backward stepwise regression based on Akaike information criteria (AIC).

\section{Results}

Basic characteristics of the study subjects are provided in Table 1.

\section{Genotype distribution of the rs1048261 SNP in HSPB7}

Observed genotype frequencies of the rs1048261 SNP in HSPB7 were in agreement with the Hardy-Weinberg equilibrium $(\mathrm{p}=0.971)$. The frequencies of the AA, AT and TT genotypes for the rs1048261 SNP were $10.0 \%, 43.2 \%$ and $46.8 \%$. Allele frequencies were $31.6 \%$ for the A allele and $68.4 \%$ for the $\mathrm{T}$ allele. No statistical differences were found in genotype distribution between genders.

\section{Relationship between two categorical variables}

Analyses of possible relationships of categorical variables (genotype, sex, clinical subtypes of psoriasis and personal history of CVD) were performed using Pearson's $c^{2}$ test. While the rs 1048261 genotypes were significantly associated with gender $(\mathrm{p}=0.05)$, Cramér's V $(\mathrm{V}=0.107)$, however, suggests a rather weaker effect. A statistically significant association $(\mathrm{p}=0.004)$ was found between the clinical subtypes of psoriasis and CVD, but Cramér's V $(\mathrm{V}=0.141)$ also suggests a rather weaker degree of association between these two variables.

\section{Intergroup comparisons}

The intergroup comparisons did not reveal significant differences between the clinical subtypes of the disease and/or cardiovascular comorbidity with respect to the distribution of the rs1048261 genotypes $(\mathrm{p}=\mathrm{NS})$. The only observed associations between cardiovascular comorbidity and clinical subtype of the disease were related to the age of disease onset and age at recruitment $(\mathrm{p}<0.001$ and $\mathrm{p}<0.001$, respectively).

\section{Logistic regression model for personal history of CVD}

For further analysis, a logistic regression model was created with personal CVD history as a dependent variable and genotypes, sex, clinical subtypes of psoriasis and age of psoriasis onset as independent variables. The model was then reduced using backward stepwise regression based on AIC. A likelihood-ratio test was used to compare the goodness of fit of these two models and no statistical difference was found. The final model indicated that only clinical subtypes of psoriasis and the age of psoriasis onset are statistically significant. Neither the full nor the reduced models confirmed a significant association between genotypes of rs 1048261 and the occurrence of CVD in patients with psoriasis. In the logistic regression modeling, no predictive role of rs1048261 genetic variability for CVD in psoriasis patients was confirmed.

\section{Discussion}

While it seems crucial to study the etiopathogenesis of the skin hallmarks of psoriasis and test appropriate treatment, less attention is given to the comorbidities of psoriasis. It is important to note that patients with psoriasis exhibit a $25 \%$ increase in relative risk of CVD independently of confounding factors, i.e. smoking, obesity, dyslipidemia hypertension and diabetes [4]. Nevertheless, to date, very little is known about the underlying mechanisms which would explain the higher prevalence of CVD in psoriasis. In this study we investigated the rs1048261 SNP within the 3'UTR of HSPB7 as an independent risk factor for CVD in psoriasis. The SNP within 3'UTR suggests the

Table 1. Basic characteristics of study subjects.

\begin{tabular}{|c|c|c|c|c|c|c|c|c|c|}
\hline & \multicolumn{4}{|c|}{ Men } & \multicolumn{4}{|c|}{ Women } & \multirow[t]{2}{*}{ Overall } \\
\hline & $\mathbf{A A}$ & AT & TT & no data & $\mathbf{A A}$ & AT & TT & no data & \\
\hline No CVD history & 87 & 65 & 12 & 10 & 93 & 84 & 24 & 15 & 390 \\
\hline Plaque psoriasis & 64 & 46 & 9 & 8 & 68 & 59 & 19 & 13 & 286 \\
\hline Pustular psoriasis & 2 & 4 & & 1 & 3 & 4 & & & 14 \\
\hline Guttate psoriasis & 21 & 15 & 3 & 1 & 22 & 21 & 5 & 2 & 90 \\
\hline CVD positive history & 31 & 30 & 4 & 5 & 32 & 45 & 12 & 9 & 168 \\
\hline Plaque psoriasis & 26 & 25 & 3 & 5 & 27 & 37 & 10 & 9 & 142 \\
\hline Pustular psoriasis & 1 & 3 & 1 & & 1 & 2 & & & 8 \\
\hline Guttate psoriasis & 4 & 2 & & & 4 & 6 & 2 & & 18 \\
\hline Overall & 118 & 95 & 16 & 15 & 125 & 129 & 36 & 24 & 558 \\
\hline
\end{tabular}


Hruska P (2016) No association of the SNP rs1048261 within 3’UTR of HSPB7 with cardiovascular morbidity in patients with psoriasis; a possible effect on miRNA-mediated translational regulation

possible involvement of the deregulated mechanism of miRNA posttranscriptional gene regulation which might be impaired with the SNP. This impairment would affect the protein levels of cardiac specific HSPB7 in cells and thus contribute to increased CVD morbidity in patients with psoriasis. We therefore investigated the possible association of the SNP rs1048261 in a large cohort of psoriasis patients $(n=558)$ where $30 \%$ of the patients had a personal history of CVD.

When we hypothesis the possible link between psoriasis and the risk of CVD, we focused on the main contributors to psoriasis pathogenesis which is the chronic inflammation and production of oxidative stress. Although both processes are closely related in pathogenesis of psoriasis, oxidative stress is responsible for cellular damages and modifications of proteins, lipoproteins and lipids. These damages may then significantly contribute the development of CVD [10,29,30]. Even though cells are equipped with variety of defense mechanisms, an impairment of such mechanism may be considered as a risk factor for CVD. One of the candidate genes with a possible role in this impairment was HSPB7, the cardiac-specific Hsp with an increase expression in response to oxidative stress [31,32]. For example, significantly elevated concentration of HSPB7 was detected in plasma of patients with acute chest pain in association with acute coronary syndrome [17].

Not only elevated protein concentration but also genetic variability within HSPB7 was previously observed in association with both ischemic and nonischemic cardiomyopathies [33,34]. Another study described SNP rs1739843 in HSPB7 in association with dilated cardiomyopathy, a structural heart disease [35]. Similarly, Villard et al. [36] in extensive GWAS of idiopathic dilated cardiomyopathy identified SNPs associated with this pathology. This was the first and only study, until now, to mention the SNP rs1048261 in association with CVD [36]. This pathological heart condition was also observed with an increased prevalence in psoriasis patients [37]. The GWAS associated the minor allele (A) of rs1048261 SNP with a reduced risk of dilated cardiomyopathy [36].

In the in silico analysis of the SNP rs1048261, several predictions of the possible effects on miRNA-mRNA interaction were found. The databases (MirSNP, miRNASNP2) we used for this analysis mostly predicted a loss of the binding site in the case of the major allele $\mathrm{T}$. Because the SNP is entered in the SNP database in a forward orientation to the genome and the coding sequence of HSPB7 is in the opposite direction, the actual breakage of the binding site is associated with the complementary base A in the mRNA of HSPB7. The SNP change may thus modify miRNA binding to HSPB7 mRNA, thereby influencing the efficiency of miRNA-mediated translational inhibition of HSPB7.

Although our results did not show any association of the SNP rs104261 genotypes with CVD in our cohort of psoriasis patients in none of the analyzed variables, i.e. gender, clinical subtypes of psoriasis, age at psoriasis onset; we still may discuss the possible effect of the SNP and associated miRNAs. Due to the fact that miRNAs have a pleiotropic character and are thus able to regulate the expression of multiple target genes, we may observe several pathways in which the miRNA could contribute to disease pathology. Therefore, when searching PubMed for the candidate miRNAs in association with cardiovascular morbidity and/or oxidative stress, several associations were found for each of them. However, only miR-15a and miR-195 were observed with a cardiovascular event frequent in psoriasis patients. MiR$15 \mathrm{a}$ was found to be downregulated in patients with acute coronary syndrome [38], whereas acute coronary syndrome belongs among the major cardiovascular events affecting psoriasis patients [39]. MiR-195 was observed as overexpressed in cardiomyocytes with an increased production of ROS and induced apoptosis. The downregulation of miR-195 decreased ROS production and apoptosis in palmitatestimulated cardiomyocytes. Sirt1, which exerts an inhibitory effect on apoptosis, was identified as a direct target [40].

The observed deregulation of these miRNAs can itself contribute to disease pathology and for this reason even small changes in this well-balanced mechanism can be crucial. In our case, the loss of bind site with the major allele $\mathrm{T}$ would cause an overexpression of HSPB7 which is observed in patients with acute chest pain [17]. On the other hand, the minor allele A would be associated with standard regulation of gene expression, which may or may not be in accordance with the GWAS study suggesting the protective character of minor allel [36]. The structural and functional character of HSPB7 suggests that overexpression could be beneficial during the cardiovascular event. Contrarily, while the standard regulation can ensure the proper regulation process, the standard version of regulation may also be involved in binding of deregulated miRNAs which may significantly change the expression of HSPB7.

Additionally to used miRNA/SNP databases, the miRdSNP database (http://mirdsnp.ccr.buffalo.edu/; accessed March 16, 2016) associates rs1048261 with two more miRNAs (miR-874, miR-143) which bind to the 3'UTR downstream from the SNP location. Both miRNA binding sites are experimentally validated and the database suggests a possible effect of the SNP rs1048261 on the regulation function of both miRNAs.

The main strength of the study is the large number of patients included in the cohort, all originating from a Central European Caucasian population. However, psoriasis development and the clinical course of the disease are both influenced by a substantial number of confounding factors, including socio-economic factors which were not investigated in this study and thus represent a possible limitation. Also, more information on the actual functional impact of the investigated polymorphism is necessary.

Even though our study did not find any association of the SNP rs1048261 genotype with CVD in patients with psoriasis, we believe that this polymorphism should be studied independently in other studies which would focus on a specific, properly diagnosed CVD. Likewise, an experimental validation of the candidate miRNAs binding with the mRNA of HSPB7 would be appreciated.

\section{Acknowledgements}

This study was supported by the CETOCOEN PLUS project and the RECETOX infrastructure, supported by the Ministry of Education, Youth and Sports of the Czech Republic (LM2011028).

\section{References}

1. Parisi R, Symmons DP, Griffiths CE, Ashcroft DM; Identification and Managemen of Psoriasis and Associated ComorbidiTy (IMPACT) project team (2013) Global epidemiology of psoriasis: a systematic review of incidence and prevalence. $J$ Invest Dermatol 133: 377-385. [Crossref]

2. Nestle FO, Kaplan DH, Barker J (2009) Psoriasis. N Engl J Med 361: 496-509. [Crossref]

3. Kimball AB, Gladman D, Gelfand JM, Gordon K, Horn EJ, et al. (2008) National Psoriasis Foundation clinical consensus on psoriasis comorbidities and recommendations for screening. J Am Acad Dermatol 58: 1031-1042. [Crossref]

4. Gaeta M, Castelvecchio S, Ricci C, Pigatto P, Pellissero G, et al. (2013) Role of psoriasis as independent predictor of cardiovascular disease: a meta-regression analysis. Int $J$ Cardiol 168: 2282-2288. [Crossref] 
Hruska P (2016) No association of the SNP rs1048261 within 3'UTR of HSPB7 with cardiovascular morbidity in patients with psoriasis; a possible effect on miRNA-mediated translational regulation

5. Gelfand JM, Neimann AL, Shin DB, Wang X, Margolis DJ, et al. (2006) Risk of myocardial infarction in patients with psoriasis. JAMA 296: 1735-1741. [Crossref]

6. Khalid U, Ahlehoff O, Gislason GH, Kristensen SL, Skov L, et al. (2014) Psoriasis and risk of heart failure: a nationwide cohort study. Eur J Heart Fail 16: 743-748. [Crossref]

7. Mehta NN, Azfar RS, Shin DB, Neimann AL, Troxel AB, et al. (2010) Patients with severe psoriasis are at increased risk of cardiovascular mortality: cohort study using the General Practice Research Database. Eur Heart J 31: 1000-1006. [Crossref]

8. Neimann AL, Shin DB, Wang X, Margolis DJ, Troxel AB, et al. (2006) Prevalence of cardiovascular risk factors in patients with psoriasis. J Am Acad Dermatol 55: 829-835. [Crossref]

9. Armstrong AW, Voyles SV, Armstrong EJ, Fuller EN, Rutledge JC (2011) Angiogenesis and oxidative stress: common mechanisms linking psoriasis with atherosclerosis. $J$ Dermatol Sci 63: 1-9. [Crossref]

10. Ferretti G, Bacchetti T, Campanati A, Simonetti O, Liberati G, et al. (2012) Correlation between lipoprotein(a) and lipid peroxidation in psoriasis: role of the enzyme paraoxonase-1. Br J Dermatol 166: 204-207. [Crossref]

11. Rocha-Pereira P, Santos-Silva A, Rebelo I, Figueiredo A, Quintanilha A, et al. (2001) Dislipidemia and oxidative stress in mild and in severe psoriasis as a risk for cardiovascular disease. Clin Chim Acta 303: 33-39. [Crossref]

12. Kalmar B, Greensmith L (2009) Induction of heat shock proteins for protection against oxidative stress. Adv Drug Deliv Rev 61: 310-318. [Crossref]

13. Krief S, Faivre JF, Robert P, Le Douarin B, Brument-Larignon N, et al. (1999) Identification and characterization of cvHsp. A novel human small stress protein selectively expressed in cardiovascular and insulin-sensitive tissues J Biol Chem. 274: 36592-36600. [Crossref]

14. Augusteyn RC (2004) alpha-crystallin: a review of its structure and function. Clin Exp Optom 87: 356-366. [Crossref]

15. Ke L, Meijering RA, Hoogstra-Berends F, Mackovicova K, Vos MJ, et al. (2011) HSPB, HSPB6, HSPB7 and HSPB8 protect against RhoA GTPase-induced remodeling in tachypaced atrial myocytes. PLoS One 6: e20395. [Crossref]

16. Yang Z, Wang Y, Lu Y, Zhao X (2011) Molecular characterization of rat cvHsp/HspB7 in vitro and its dynamic molecular architecture. Mol Med Rep 4: 105-111. [Crossref]

17. Chiu TF, Li CH, Chen CC, Chen CH, Cheng CJ, et al. (2012) Association of plasma concentration of small heat shock protein B7 with acute coronary syndrome. Circ J 76: 2226-2233. [Crossref]

18. Bartel DP1 (2004) MicroRNAs: genomics, biogenesis, mechanism, and function. Cell 116: 281-297. [Crossref]

19. Lewis BP, Shih IH, Jones-Rhoades MW, Bartel DP, Burge CB (2003) Prediction of mammalian microRNA targets. Cell 115: 787-798. [Crossref]

20. Joyce CE, Zhou X, Xia J, Ryan C, Thrash B, et al. (2011) Deep sequencing of smal RNAs from human skin reveals major alterations in the psoriasis miRNAome. Hum Mol Genet 20: 4025-4040. [Crossref]

21. Sonkoly E, Stahle M, Pivarcsi A (2008) MicroRNAs: novel regulators in skin inflammation. Clin Exp Dermatol 33: 312-315. [Crossref]

22. Small EM, Olson EN (2011) Pervasive roles of microRNAs in cardiovascular biology. Nature 469: 336-342. [Crossref]

23. Wang GK, Zhu JQ, Zhang JT, Li Q, Li Y, et al. (2010) Circulating microRNA: a novel potential biomarker for early diagnosis of acute myocardial infarction in humans. Eur Heart J 31: 659-666. [Crossref]
24. Gong J, Tong Y, Zhang HM, Wang K, Hu T, et al. (2012) Genome-wide identification of SNPs in microRNA genes and the SNP effects on microRNA target binding and biogenesis. Hum Mutat 33: 254-263. [Crossref]

25. Sun G, Yan J, Noltner K, Feng J, Li H, et al. (2009) SNPs in human miRNA genes affect biogenesis and function. RNA 15: 1640-1651. [Crossref]

26. Griffiths CE, Christophers E, Barker JN, Chalmers RJ, Chimenti S, et al. (2007) A classification of psoriasis vulgaris according to phenotype. Br J Dermatol 156: 258262. [Crossref]

27. Liu C, Zhang F, Li T, Lu M, Wang L, et al. (2012) MirSNP, a database of polymorphisms altering miRNA target sites, identifies miRNA-related SNPs in GWAS SNPs and eQTLs. BMC Genomics 13: 661. [Crossref]

28. Gong J, Liu C, Liu W, Wu Y, Ma Z, et al. (2015) An update of miRNASNP database for better SNP selection by GWAS data, miRNA expression and online tools. Database (Oxford) 2015: bav029. [Crossref]

29. Kaur S, Zilmer K, Leping V, Zilmer M (2013) Serum methylglyoxal level and it association with oxidative stress and disease severity in patients with psoriasis. Arch Dermatol Res 305: 489-494. [Crossref]

30. Kaur S, Zilmer K, Leping V, Zilmer M (2011) The levels of adiponectin and leptin and their relation to other markers of cardiovascular risk in patients with psoriasis. $J$ Eur Acad Dermatol Venereol 25: 1328-1333. [Crossref]

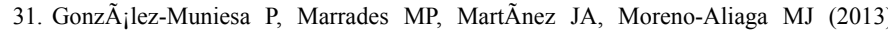
Differential Proinflammatory and Oxidative Stress Response and Vulnerability to Metabolic Syndrome in Habitual High-Fat Young Male Consumers Putatively Predisposed by Their Genetic Background. Int J Mol Sci 14: 17238-17255. [Crossref]

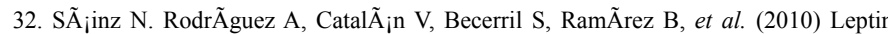
Administration Downregulates the Increased Expression Levels of Genes Related to Oxidative Stress and Inflammation in the Skeletal Muscle of ob/ob Mice. Mediator Inflamm 2010: e784343.

33. Cappola TP, Li M, He J, Ky B, Gilmore J, et al. (2010) Common variants in HSPB7 and FRMD4B associated with advanced heart failure. Circ Cardiovasc Genet 3: 147154. [Crossref]

34. Matkovich SJ, Van Booven DJ, Hindes A, Kang MY, Druley TE, et al. (2010) Cardiac signaling genes exhibit unexpected sequence diversity in sporadic cardiomyopathy, revealing HSPB7 polymorphisms associated with disease. J Clin. Invest 120: 280-289. [Crossref]

35. Stark K, Esslinger UB, Reinhard W, Petrov G, Winkler T, et al. (2010) Genetic Association Study Identifies HSPB7 as a Risk Gene for Idiopathic Dilated Cardiomyopathy. PLoS Genet 6: e1001167. [Crossref]

36. Villard E, Perret C, Gary F, Proust C, Dilanian G, et al. (2011) A genome-wide association study identifies two loci associated with heart failure due to dilated cardiomyopathy. Eur Heart J 32: 1065-1076. [Crossref]

37. Eliakim-Raz N, Shuvy M, Lotan C, Planer D (2008) Psoriasis and dilated cardiomyopathy: coincidence or associated diseases? Cardiology 111: 202-206. [Crossref]

38. Liu X, Wang L, Li H, Lu X, Hu Y (2014) Coactivator-associated arginine methyltransferase 1 targeted by miR-15a regulates inflammation in acute coronary syndrome. Atherosclerosis 233: 349-356. [Crossref]

39. Parisi R, Rutter MK, Lunt M, Young HS, Symmons DP (2015) Psoriasis and the Risk of Major Cardiovascular Events: Cohort Study Using the Clinical Practice Research Datalink. J Invest Dermatol 135: 2189-2197. [Crossref]

40. Zhu H, Yang Y, Wang Y, Li J, Schiller PW, et al. (2011) MicroRNA-195 promotes palmitate-induced apoptosis in cardiomyocytes by down-regulating Sirt1. Cardiovasc Res 92: 75-84. [Crossref]

Copyright: (C2016 Hruska P. This is an open-access article distributed under the terms of the Creative Commons Attribution License, which permits unrestricted use, distribution, and reproduction in any medium, provided the original author and source are credited. 